\title{
La pasión por el trabajo, ¿vino viejo en botella nueva?: Un análisis psicométrico de la Escala de Pasión por el Trabajo de Vallerand et al.
}

\author{
Carla Ortega Santori ${ }^{1}$, Miguel Martínez-Lugo², Ramón Rodríguez Montalbán ${ }^{3}$ \\ Recibido: 15/06/2017 Aceptado: 19/09/2017 \\ DOI: 10.21772/ripo.v35n2a02
}

\begin{abstract}
Resumen
El objetivo de este estudio es determinar las propiedades psicométricas de la Escala de Pasión por el Trabajo de Vallerand et al. (2003) traducida por Orgambidez-Ramos et al. (2014) y diferenciar el concepto de pasión por el trabajo de engagement en el trabajo, la centralidad del trabajo, la motivación intrínseca y el significado del trabajo. La muestra estuvo compuesta por 1,016 personas de diversos sectores laborales trabajando en Puerto Rico. Nuestros resultados confirman la estructura bi-factorial de la escala (pasión armoniosa y pasión obsesiva) con niveles de confiabilidad aceptables. Concluimos que la escala es un instrumento adecuado para el uso en el contexto laboral puertorriqueño y que la pasión por el trabajo es una aportación única a la literatura académica de la psicología ocupacional.
\end{abstract}

Palabras clave: Pasión por el Trabajo, Análisis Psicométrico, Validez Discriminante.

\section{The Passion for Work ¿old wine in new bottle? Psychometric properties of the Passion for Work Scale of Vallerand et al.}

\begin{abstract}
The purpose of this study is to determine the psychometric properties of the Passion for Work Scale of Vallerand et al. (2003) translated to Spanish by Orgambídez-Ramos et al. (2014); and to differentiate passion for work from work engagement, work centrality, intrinsic motivation, and meaning of work. The sample consisted of 1,016 participants from various industries working in Puerto Rico. Our results indicate that the two-factor structure (harmonious passion and obsessive passion) is the most appropriate and has adequate reliability measures. The Passion for Work Scale is an adequate instrument for use in the Puerto Rican context and passion for work is a unique contribution to the academic literature of occupational psychology.
\end{abstract}

Key words: Passion for Work, Psychometric Analysis, Discriminant Aalidity.

\footnotetext{
1 Carla Ortega Santori-Doctora. Departamento de Psicología Industrial Organizacional. Universidad Carlos Albizu. San Juan. Puerto Rico. Email: carla. ortega8@gmail.com

2 Miguel Martínez Lugo-Doctor. Departamento de Psicología Industrial Organizacional. Director. Universidad Carlos Albizu. San Juan. Puerto Rico. Email: mmartinez@albizu.edu

3 Ramon Rodríguez Montalbán-Doctor. Departamento de Psicología Industrial Organizacional. Profesor. Universidad Carlos Albizu. San Juan. Puerto Rico. Email: rmontalban@albizu.edu
}

Nota: El presente artículo fue publicado en el 2017. Sin embargo, con el fin de mantener la periodicidad de la Revista, el número corresponde al segundo semestre del año 2016.

Cómo citar este artículo: Ortega Santori, C., Martínez Lugo, M. \& Rodríguez Montalbán, R. (2016). La pasión por el trabajo, ¿ vino viejo en botella nueva?: Un análisis psicométrico de la Escala de Pasión por el Trabajo de Vallerand et al. Revista Interamericana de Psicología Ocupacional, 35(2), 68-79. DOI: $10.21772 /$ ripo.v35n2a02 


\section{Introducción}

Hace 20 años la compañía Apple se encontraba en un momento crítico con un declive en sus ingresos de 30\% y pérdidas de $\$ 161$ millones en un trimestre (Liu, 1997). Steve Jobs regresa a la compañía en el mismo año como principal ejecutivo y lleva a cabo un proceso de re-estructuración que los ayuda a convertirse en una de las principales compañías de tecnología en el mercado. En el lanzamiento de esta nueva fase de la compañía, Jobs dice en una conferencia, "Nuestro valor principal [en Apple] es que creemos que las personas con pasión pueden cambiar el mundo para bien." Jobs no es el único que sostiene que la pasión es un elemento importante para no solo ser exitoso, sino que para prosperar en el trabajo (Duckworth, 2016). Igualmente, el interés público por la pasión por el trabajo ha aumentado durante la pasada década, lo cual se ve reflejado en el número de búsquedas del término (Google, 2017). De igual manera, la literatura académica refleja el interés ascendiente en el tema. Una búsqueda en EBSCOhost en abril de 2017 con las palabras clave "pasión por el trabajo" y "passion for work" obtuvo como resultado 649,153 publicaciones científicas.

A pesar de la gran cantidad de investigaciones que se ha generado sobre la pasión por el trabajo, existe confusión con otros conceptos similares propios de la psicología ocupacional (Perrewé, Hochwarter, Ferris, Mcallister \& Harris, 2014). Un ejemplo claro de esta polémica es el libro publicado por algunos de los exponentes principales del engagement en el trabajo, Salanova y Schaufeli (2009), El engagement en el trabajo: cuando el trabajo se convierte en pasión. En el título hacen referencia a los posibles traslapos que existen entre la pasión y el engagement. Sin embargo, para aportar a la literatura de psicología ocupacional es necesario establecer las distinciones conceptuales entre la pasión y constructos similares. Por lo tanto, nos preguntamos, ¿La pasión por el trabajo es un constructo distinto al engagement en el trabajo, la centralidad del trabajo, la motivación intrínseca y el significado del trabajo? Además y desde la perspectiva de medición del constructo, ¿cuáles son las propiedades psicométricas de la Escala de Pasión por el Trabajo de Vallerand et al. (2003) en una muestra de personas que se encuentran trabajando en Puerto Rico?

Esta investigación tiene como propósito determinar las propiedades psicométricas de la Escala de Pasión por el Trabajo en una muestra de trabajadores en Puerto Rico. Además, se examina la validez discriminante de la pasión por el trabajo utilizando como variables criterio el engagement en el trabajo, la motivación intrínseca, la centralidad en el trabajo y el significado del trabajo. El estudio ofrece respuestas a la pregunta, ¿la pasión por el trabajo es vino viejo en botella nueva?

\section{Pasión por el trabajo}

La Teoría de la Autodeterminación de Deci y Ryan (1985) explica cómo una actividad se convierte en pasión. Según la teoría, las personas tienen una inclinación innata de internalizar ciertas actividades en su auto-concepto. De acuerdo al nivel de importancia, el valor y las consonancias que existan con el self, se puede integrar la actividad en la identidad de la persona. De esta forma, si la persona llega a identificarse con el valor de la actividad y esta ocupa una parte central en su identidad, el comportamiento se vuelve auto-determinado y la actividad se convierte en una pasión (Vallerand et al., 2003).

Los autores citados proponen un modelo dual para explicar la pasión y definirla como una inclinación fuerte hacia una actividad que la persona encuentra importante, que le gusta, que es parte de la identidad de la persona y a la cual le dedica tiempo y energía. Además, establecen que la pasión puede ser experimentada de dos formas: obsesiva y armoniosamente. Para esta conceptuación, los investigadores se basan en los diferentes acercamientos filosóficos que existen de la pasión: el que la define como una fuerza que se apodera de la razón y el que la define como una fuerza de impulso que coexiste con la razón. En la misma línea de esta base filosófica, el modelo dual de pasión define la pasión obsesiva como aquella que entra en conflicto con algunos aspectos del yo y otras actividades en la vida de la persona, llevando a unos resultados menos adaptativos. Por otro lado, la pasión armoniosa es aquella que existe en consonancia con otros aspectos del yo y actividades, llevando a unos resultados adaptativos. El tipo de internalización que se lleve a cabo determinará si la pasión es armoniosa u obsesiva (Vallerand et al., 2003).

La pasión armoniosa surge de un proceso de internalización integrada. Las personas que logran este tipo de internalización identifican la importancia de la actividad y lo integran en su auto-concepto. Una persona en un ambiente donde se propicia la autonomía, es más propensa a desarrollar una pasión armoniosa porque no se generan contingencias adscritas al trabajo (Mageau et al., 2009). Si la persona logra satisfacer las necesidades de autonomía, competencia y relación, se lleva a cabo una integración coherente donde los comportamientos son impulsados por el deseo o disfrute de la persona (Deci \& Ryan, 2000; Mageau et al., 2009). Es por eso que las personas apasionadas armoniosamente logran controlar su impulso de involucrarse en el trabajo (Deci \& Ryan, 2000; Vallerand et al., 2003). La motivación intrínseca desarrollada a raíz de este proceso produce comportamientos auto-determinados que no necesitan refuerzos porque son impulsados por el deseo o el disfrute del trabajo. 
Por otro lado, la pasión obsesiva surge de un proceso de internalización controlada. Es decir, el individuo no logra integrar completamente la actividad a su auto-concepto y esto entra en conflicto con otras partes de su identidad. El comportamiento socializado de participar en la actividad se debe a las contingencias adscritas como: la auto-estima, el orgullo, la culpa y/o la vergüenza. Ya que la actividad no satisface las necesidades de competencia, autonomía y relación, el comportamiento resultante depende de las contingencias externas explicadas, como no sentirse aceptado 4 o culpable por no haber participado en la actividad (Deci, Eghrari, Patrick \& Leon, 1994; Deci \& Ryan, 2000). Por lo tanto, las personas con una pasión obsesiva suelen adscribir el sentido de ego y auto-estima a su identificación con el trabajo. Como resultado, tienden a ser inflexibles ya que sienten un impulso abrumador de dedicar tiempo al trabajo y hacerlo a pesar de las condiciones negativas (Deci et al., 1994; Deci \& Ryan, 2000).

Los factores contextuales que influyen en el desarrollo de la pasión se han investigado para entender por qué algunas personas se apasionan armoniosamente $\mathrm{u}$ obsesivamente. Mageau et al. (2009) encontraron que las personas son más propensas a desarrollar una pasión armoniosa si personas cercanas con autoridad apoyan su autonomía. En cambio, las personas son más propensas a desarrollar una pasión obsesiva si esas mismas relaciones no permiten la autonomía y ejercen control sobre la decisión de participar en la actividad. Estos resultados concuerdan con la teoría de auto-determinación, la cual expone que la internalización depende en gran parte del contexto social. Factores contextuales tales como reconocer los sentimientos de la persona hacia la actividad, proveer razones de porqué se debería llevar a cabo la actividad y tener la opción de participar en la actividad son predictores del proceso de internalización (Deci et al.,1994; Deci \& Ryan, 2000).

\section{Conceptos similares}

Existe una necesidad de diferenciar los constructos organizacionales relacionados a la pasión para que se facilite la investigación sistemática, el desarrollo de intervenciones adecuadas y para preservar la parsimonia por la que se debe regir toda teoría científica (Le, Schmidt, Harter \& Lauver, 2010; Perrewé et al., 2014; Salanova \& Schaufeli, 2009). La psicología positiva se ha encontrado con algunas críticas hacia la falta de rigurosidad metodológica, ideológica y conceptual de la investigación (Hackman, 2009). Esencialmente, se acusa a la literatura de la psicología positiva de embotellar vino viejo en botella nueva (Fernández-Ríos, 2008; FernándezRíos \& Novo, 2012; Lazarus, 2003; Pérez-Álvarez, 2012).

Nota: Para facilitar la lectura, los autores han optado por hacer uso del género masculino para referirse a personas de ambos géneros (masculino y femenino).
Por tal motivo, es esencial establecer la independencia de la pasión por el trabajo de otros conceptos similares como son el engagement en el trabajo, la centralidad del trabajo, la motivación intrínseca y el significado del trabajo. Una diferencia fundamental entre la pasión por el trabajo y el resto de los conceptos es la naturaleza dual del primero donde existen dos tipos: la pasión obsesiva y la pasión armoniosa (Vallerand et al., 2003).

La pasión se ha comparado con constructos de estado mental como el engagement en el trabajo (Perrewé et al., 2014). Desde el marco teórico de la salud ocupacional, el engagement en el trabajo es el estado mental positivo relacionado al trabajo que se caracteriza por el vigor, la dedicación y la absorción (Schaufeli, Salanova, GonzálezRoma \& Bakker, 2002). Tanto la pasión armoniosa como el engagement son estados positivos que incluyen un proceso motivacional por el cual se satisfacen las necesidades intrínsecas de un empleado, y ambos conceptos se caracterizan por los altos niveles de energía y persistencia. Sin embargo, se diferencian en componentes esenciales. En primera instancia, el engagement en el trabajo es un constructo afectivo y cognitivo que representa un estado mental. Por otro lado, la pasión por el trabajo es un constructo motivacional desarrollado a partir de una internalización, por lo que se considera parte de la identidad de la persona. Además, se diferencian en el grado que forman parte de la identidad de la persona. Por un lado, la pasión ocupa un nivel central en la identidad general de la persona. Mientras que el engagement se refiere a un estado de entusiasmo, persistencia mental y concentración en el trabajo exclusivamente (Salanova \& Schaufeli, 2009; Vallerand et al., 2003). Incluso, se ha encontrado que la pasión armoniosa actúa como mediadora entre los recursos laborales y la dimensión de vigor del engagement en el trabajo (Trépanier et al., 2014). La pasión armoniosa actúa como antecedente del engagement, ya que propicia una realización flexible de las tareas que permite una absorción cognitiva.

De igual forma, existen algunas similitudes entre el significado del trabajo y la pasión por el trabajo. Según el Meaning of Working (MOW) International Research Team (1987) el significado del trabajo es la interpretación individual de qué significa o qué representa el trabajo en el contexto general de la vida de un individuo. Wrzesniewski, Dutton y Debebe (2003) indicaron que existe una influencia interpersonal en el desarrollo del significado del trabajo a través de las señales que otros ofrecen en relación al valor del trabajo. El significado del trabajo y la pasión por el trabajo se nutren de las experiencias positivas vividas en el trabajo y el valor adscrito a este (Mageau et al., 2009; Wrzesniewski et al., 2003). En ambos procesos psicológicos las personas construyen actitudes hacia el trabajo partiendo 
de las interacciones sociales y la interpretación de información. Sin embargo, el significado del trabajo no incluye el impulso o inclinación fuerte hacia el trabajo que distingue a la pasión.

Otro concepto parecido a la pasión es la centralidad del trabajo o el nivel de importancia y valor que tiene el trabajo, de forma general, en la vida de un individuo (MOW, 1987; Paullay, Alliger \& Stone-Romero, 1994). En ambos constructos está presente la comparación que un individuo realiza entre el trabajo y otros intereses en su vida para de esa forma establecer su valor. Además, en ambos vemos la influencia del proceso de socialización del individuo (Ashforth \& Mael, 1989; Mageau et al., 2009). Las personas apasionadas obsesivamente por su trabajo y las que lo consideran un aspecto central en su vida le asignan un alto nivel de importancia o prioridad al trabajo. No obstante, la pasión requiere además que sea una actividad que le guste al individuo, que sea parte de su identidad y que le dedique tiempo y energía. Además, la pasión por el trabajo surge de un proceso de internalización que puede llevar a resultados adaptativos (pasión armoniosa) o maladaptativos (pasión obsesiva) (Vallerand et al., 2003). La centralidad no hace distinción de dos formas de experimentarla (Paullay et al., 1994).

Al examinar constructos motivacionales, se ha identificado cierta convergencia entre la motivación intrínseca y la pasión por el trabajo (Vallerand et al., 2003). Lisbona-Bañuelos, Palací-Descals y Bernabé-Castaño (2016) encontraron que entre el 2010 y el 2015 se publicaron 20 artículos que estudian la motivación y la pasión en el contexto laboral. Según Deci y Ryan (1985), la motivación intrínseca se refiere al impulso de participar en una actividad por el disfrute y satisfacción relacionado a ella. Tanto la motivación intrínseca como la pasión sugieren un amor por la actividad, por el placer y por el valor inherente que se experimenta (Deci \& Ryan, 1985; Vallerand et al., 2003). A diferencia de la motivación intrínseca, la pasión conlleva un proceso de internalización donde se integra el trabajo como parte de la identidad del individuo (Vallerand et al., 2003). Por otro lado, la motivación intrínseca no suele ser internalizada a la identidad de la persona y surge de una interacción a corto plazo con la actividad (Deci \& Ryan, 1985; Koestner \& Losier, 2002).

\section{Variables relacionadas a la pasión por el trabajo}

A pesar de las diferencias entre la pasión armoniosa y la obsesiva, ambos tipos guardan una relación similar con los criterios incluidos en la definición de pasión. Vallerand et al. (2003) encontraron que ambos tipos de pasión ven la actividad como una pasión, la consideran importante o de mucho valor, invierten tiempo y energía en la misma y la consideran parte de su identidad. Solo la pasión obsesiva se asocia significativamente con el conflicto con otras actividades (Vallerand et al., 2003).

En el contexto organizacional, se ha estudiado el efecto que tiene la pasión por el trabajo, encontrándose que la pasión armoniosa correlaciona positivamente con variables como flow, concentración (Vallerand et al., 2003; Forest, Mageau, Sarrazin \& Morin, 2011), bienestar psicológico, compromiso afectivo, vitalidad (Forest et al., 2011), alto desempeño (Ho, Wong \& Lee, 2011), satisfacción de vida (Birkeland \& Buch, 2014), mayor percepción de apoyo y control, menor percepción de sobrecarga (Lavigne, Forest, Fernet \& Crevier-Braud, 2014) y menos burnout (Vallerand et al., 2010). Por otro lado, se ha encontrado que la pasión obsesiva correlaciona con el malestar psicológico, afecto negativo, persistencia rígida (Vallerand et al., 2003; Forest et al., 2011), mayor percepción de sobrecarga (Lavigne et al., 2014) y mayor burnout (Birkeland \& Buch, 2014; Vallerand et al., 2010).

\section{Medición de la pasión}

La Escala de Pasión de Vallerand et al. (2003) es, hasta el momento, el instrumento más utilizado, para medir la pasión por el trabajo (Curran, Hill, Appleton, Vallerand \& Standage, 2015). Los autores desarrollaron la escala inicialmente para medir pasión por una actividad en términos generales. Esta escala ha sido adaptada para el trabajo y se ha utilizado en varias investigaciones para estudiar la relación de la pasión con otras variables psicosociales y organizacionales (Marsh et al., 2013). Las premisas de la escala han sido adaptadas para hacer referencia directa al trabajo (Por ejemplo, "Las cosas nuevas que aprendo con este trabajo hacen que lo valore todavía más").

El estudio original de las propiedades psicométricas de la Escala de Pasión (Vallerand et al., 2003) se realizó con cuatro muestras independientes de estudiantes universitarios $(n=539)$, atletas universitarios varones $(n=205)$, ciclistas $(n=59)$ y apostadores de juegos al azar $(n=146)$. En todas las muestras el modelo bi-factorial mostró tener mejor ajuste y la fiabilidad de las sub-escalas fue aceptable. La escala original consistía de 34 ítems y fue reducida a 14 de acuerdo a los resultados del análisis exploratorio de factores (Vallerand et al., 2003). En estudios subsiguientes se han utilizado versiones reducidas de 6 y 12 ítems (Marsh et al., 2013).

Hasta el momento, la Escala de Pasión de Vallerand et al. (2003) se ha utilizado en investigaciones en Canadá (Donahue et al., 2012), Francia (Vallerand et al., 2010), Suecia (Thorgren, Wincent \& Sirén, 2013), Italia (Zito \& Colombo, 2017), Rusia (Astakhova, 2015), Noruega (Donahue et al., 2012) y España (Chamarro et al., 2015). 
De igual manera, se ha administrado en francés (Vallerand et al., 2003), inglés (Vallerand \& Houlfort, 2003), ruso (Astakhova, 2015) y español (Chamarro et al., 2015; Orgambídez-Ramos, Borrego-Alés y Gonçalves, 2014). Además, la Escala de Pasión se ha utilizado para evaluar los niveles de pasión por una variedad de actividades, tales como estudios, música (Mageau et al., 2009), deportes, juegos de azar (Vallerand et al., 2003) y el trabajo (Vallerand \& Houlfort, 2003).

En una investigación realizada por Marsh et al. (2013), se estudió la estructura factorial de la escala a través de 19 muestras con un total de 3,570 participantes. Los autores confirmaron la estructura bi-factorial de la escala, la cual es invariante por género y lenguaje. Orgambídez-Ramos et al. (2014) tradujeron la Escala de Pasión por el Trabajo al español utilizando el método de traducción inversa. El proceso resultó en una Escala de Pasión por el Trabajo compuesta por 14 ítems, se reprodujo la estructura bifactorial y las sub-escalas obtuvieron índices de fiabilidad superior a .90 .

\section{Propósito del estudio}

El estudio de la pasión por el trabajo ha estado creciendo a través de los años. Hasta el momento, no ha sido estudiada en el contexto laboral de Puerto Rico. Por lo tanto, es nuestro interés analizar las propiedades psicométricas de la Escala de Pasión por el Trabajo de Vallerand et al. (2003) traducida por Orgambídez-Ramos et al. (2014) en una muestra de personas que están trabajando en Puerto Rico. Específicamente, se examina la estructura factorial a través de un análisis de factores confirmatorio, se lleva a cabo un análisis de reactivos y se analizan los indicies de confiabilidad de las sub-escalas del instrumento. Además, se evalúa validez discriminante mediante el análisis de varianza media extraída, distinguiendo la pasión por el trabajo de otros constructos tales como el engagement en el trabajo, la centralidad del trabajo, la motivación intrínseca y el significado del trabajo como variables criterio.

\section{Método}

\section{Participantes}

Se llevó a cabo un análisis secundario de los datos obtenidos de una investigación realizada por RodríguezMontalbán et al. (2016). En dicho estudio, los participantes fueron contactados por redes sociales, correo electrónico y de forma individual. A los mismos se le garantizaron sus derechos como participantes de investigación cumpliendo así con los requisitos establecidos por el Código de ética de la profesión en Puerto Rico. De la totalidad de la muestra, 501 participantes completaron los cuestionarios a través de la plataforma QuestionPro ${ }^{\circledR}$ y el restante 515 respondió en papel y lápiz. La muestra fue obtenida por disponibilidad y estuvo compuesta por 1,016 empleados, de los cuales $65 \%$ eran mujeres y $35 \%$ eran hombres. Las edades fluctuaron entre los 21 y 78 años con un promedio de 36.02 años $(D E=10.97)$. El $39 \%$ de la muestra indicó poseer un bachillerato o licenciatura. El número de años trabajando fluctuó desde menos de un año hasta 54 años, con un promedio de 12.44 años $(D E=10.51)$. La mayoría de los participantes indicó que trabaja en una organización privada (69.4\%). En cuanto al tipo de industria, el $22 \%$ se ubica en el área de servicio. La mayoría de las personas (78.2\%) informó que tiene un empleo a tiempo completo. Por último, un $71.2 \%$ indicó que no realiza tareas de supervisión.

\section{Instrumentos}

En la investigación se utilizaron los siguientes instrumentos:

Hoja de datos demográficos. Con esta hoja se solicitó información referente al género sexual, la edad, la preparación académica, el número de años trabajando, el tipo de organización donde trabaja, el sector de industria para la cual trabaja, si realiza tareas de supervisión y el tipo de jornada que lleva a cabo (a tiempo completo o a tiempo parcial).

Escala de pasión por el trabajo. La escala fue elaborada por Vallerand et al. (2003) y consta de 14 ítems distribuidos en dos sub-escalas: la pasión armoniosa (ítems 1-7) y la pasión obsesiva (ítems 8-14). Se responden en un formato tipo Likert que fluctúa desde 1 (totalmente en desacuerdo) a 7 (totalmente de acuerdo). Un ejemplo de un ítem de la sub-escala de pasión armoniosa es, "Este trabajo está en armonía con otras actividades de mi vida". Un ejemplo de un ítem de la sub-escala de pasión obsesiva es, "Mi estado de ánimo depende de mi capacidad para realizar este trabajo". En el estudio de Orgambidez-Ramos et al. (2014) el índice de fiabilidad alfa de Cronbach para la sub-escala de pasión armoniosa es de .92 y para la pasión obsesiva es de .94 .

Escala breve de centralidad del trabajo. Esta escala fue elaborada por Martínez-Lugo, Rodríguez-Montalbán y Andújar (2015) y cuenta con ocho ítems que se responden en un formato de respuesta tipo Likert que fluctúa desde 1 (totalmente en desacuerdo) a 7 (totalmente de acuerdo). Un ejemplo de ítem de esta escala es "La importancia de una persona se mide por el trabajo que realiza". Al evaluar su confiabilidad en el presente estudio, se obtuvo un coeficiente alfa de Cronbach de .87. Un análisis de factores confirmatorios con ecuaciones estructurales indicó que es una escala unifactorial con una carga mayor a .70 .

Escala de engagement. La versión corta de la Escala de Engagement en el Trabajo de Utrecht (UWES-9) fue desarrollada por Schaufeli, Bakker y Salanova (2006) y consta de nueve ítems que se responden en una escala 
tipo Likert de siete anclajes que fluctúa desde 1 (nunca o ninguna vez) hasta 7 (siempre o todos los días). Un ejemplo de ítem de esta escala es "Me siento feliz cuando estoy trabajando intensamente". De acuerdo a los análisis en este estudio, los índices de fiabilidad alfa de Cronbach para cada dimensión de la escala son los siguientes: vigor $(\alpha=.88)$, dedicación $(\alpha=.91)$ y absorción $(\alpha=.75)$. Un análisis de factores confirmatorio indicó que la escala consta de tres factores con índices de ajuste aceptables.

Escala de motivación intrínseca. La Sub-Escala de Motivación Intrínseca de Situational Motivation Scale (SIMS) de Guay, Vallerand y Blanchard (2000) consta de cuatro ítems que se responden en una escala tipo Likert de siete anclajes que fluctúa desde 1 (totalmente en desacuerdo) a 7 (totalmente de acuerdo). Cada ítem representa una razón por la que una persona realiza el trabajo. Un ejemplo de un ítem es, "Porque creo que es interesante". Al evaluar la confiabilidad de la sub-escala en el presente estudio, se obtuvo un coeficiente alfa de Cronbach de .88. Un análisis de factores de máxima verosimilitud indicó que la subescala es unifactorial con cargas factoriales mayor a .70.

Escala de significado del trabajo. La Escala del Significado de la Tarea desarrollada por Rodríguez Montalbán, Martínez Lugo y Sánchez Cardona (2015) consta de tres ítems que se responden en una escala tipo Likert con siete anclajes de respuesta que fluctúa desde 0 (totalmente en desacuerdo) a 6 (totalmente de acuerdo). Un ejemplo de un ítem es, "Mi trabajo es muy significativo". Al evaluar su confiabilidad en el presente estudio, se obtuvo un coeficiente alfa de Cronbach de .75. Un análisis de factores confirmatorio con ecuaciones estructurales indicó que es una escala unifactorial con una carga mayor a .70 .

\section{Procedimientos}

En este estudio se utilizaron datos secundarios provenientes de la investigación realizada por RodríguezMontalbán et al. (2016). Para realizar dicho estudio, los autores contaron con la aprobación del comité de revisión institucional de la Universidad Carlos Albizu de San Juan, Puerto Rico que vela porque los procedimientos cumplan con los requisitos necesarios para salvaguardar los derechos de los participantes de una investigación. Para realizar esa investigación los autores contaron con la aprobación del comité de revisión institucional de la Universidad Carlos Albizu de San Juan, Puerto Rico. Se invitó a las personas a participar del estudio completando los cuestionarios en línea y en lápiz y papel. En todo momento se garantizó la participación voluntaria, el anonimato y el derecho a abandonar el estudio cuando lo consideraran necesario sin ser penalizados.
Para propósitos de la actual investigación, se obtuvo el permiso de los investigadores para utilizar los datos recopilados. Luego de obtener el permiso de los investigadores, éstos fueron compilados en los programas SPSS versión 23, AMOS 23 y R.

Procedimientos para el análisis de datos.Los análisis estadísticos realizados fueron: estimación de la validez a través del análisis de factores confirmatorio, análisis de reactivos, estimación de confiabilidad utilizando el coeficiente alfa de Cronbach y análisis de varianza media extraída. En primer lugar, se utilizó un análisis de factores confirmatorio (AFC) para evaluar la validez de constructo de la escala. Para realizar el AFC se utilizó el programa AMOS 23 con el método de estimación de máxima verosimilitud y el programa $R$ para realizar las correcciones de Satorra y Bentler (2001) de la bondad de ajuste para datos no normales. Luego de completar el análisis factorial de la escala, se realizó un análisis de discriminación de ítems utilizando la técnica de correlación ítem-total. Además, se calculó la consistencia interna de la escala utilizando el coeficiente alfa de Cronbach.

Por último, se procedió a examinar la varianza media extraída de las escalas como recomiendan Fornell y Larcker (1981). En la presente investigación, se analizó la validez discriminante de la Escala de Pasión por el Trabajo (Vallerand et al., 2003) con la Escala Breve de Centralidad del Trabajo (Martínez Lugo et al., 2015), la Escala de Engagement en el Trabajo de Utrecht (Schaufeli et al., 2006), la Sub-Escala de Motivación Intrínseca de SIMS (Guay et al., 2000) y la Escala del Significado de la Tarea (Rodríguez Montalbán et al., 2015).

\section{Resultados}

\section{Análisis descriptivos de los ítems}

Se calcularon las medias y desviaciones estándar para los 12 ítems de la Escala de Pasión por el Trabajo de Vallerand et al. (2003) para analizar las propiedades de distribución de la misma. Las medias de los ítems fluctuaron entre 2.16 y 5.32, mientras que las desviaciones estándar fluctuaron entre .05 y .52 . Los estadísticos Kolmogorov-Smirnov y Shapiro-Wilk ofrecen evidencia empírica que los datos no siguen una distribución normal (véase Tabla 1). Se realizó el análisis de normalidad multivariante de los datos mediante las pruebas estadísticas $M$ de Mardia, Doornik-Hansen y Henze-Zirkler (Doornik \& Hansen, 2008).

Los resultados muestran que no hay normalidad multivariante de los datos: $M$ de asimetría $=27.95, X^{2}(560)$ $4557.89, p<.001, M$ de curtosis $=345.29, X^{2}(1) 8005.28, p$ $<.001$, Henze-Zirkler $=3.76, X^{2}$ (1) 4.30, $p<.001$, Doornik- 
Hansen $=X^{2}$ (28) 1461.59, $p<.001$. La no no-normalidad de los datos justifica el uso de las correcciones de Satorra y Bentler (2001) para calcular el ajuste de los modelos de ecuaciones estructurales. Las correcciones han sido utilizadas en la presente investigación para evaluar el ajuste de los modelos de ecuaciones estructurales.

Tabla 1: Estadísticos descriptivos y de distribución de los items de la Escala de Pasión por el Trabajo

\begin{tabular}{ccccccc}
\hline Ítem & Media & $\begin{array}{c}\text { Desviación } \\
\text { Estándar }\end{array}$ & Asimetría & Curtosis & $\begin{array}{c}\text { Kolmogorov- } \\
\text { Smirnov }\end{array}$ & $\begin{array}{c}\text { Shapiro- } \\
\text { Wilk }\end{array}$ \\
\hline PA1 & 5.32 & .05 & -.89 & .48 & .18 & .87 \\
PA2 & 5.29 & .48 & -.79 & .32 & .16 & .89 \\
PA3 & 5.30 & .05 & -.80 & .29 & .17 & .88 \\
PA4 & 5.19 & .05 & -.72 & .09 & .15 & .89 \\
PA5 & 4.86 & .05 & -.54 & -.30 & .14 & .91 \\
PA6 & 4.61 & .05 & -.33 & -.56 & .14 & .92 \\
PA7 & 4.82 & .52 & -.46 & -.32 & .13 & .92 \\
PO1 & 2.98 & .05 & .54 & -.42 & .18 & .89 \\
PO2 & 3.05 & .05 & .54 & -.39 & .17 & .89 \\
PO3 & 2.59 & .05 & .81 & .03 & .24 & .84 \\
PO4 & 2.52 & .05 & .96 & .14 & .25 & .82 \\
PO5 & 2.53 & .05 & .85 & .14 & .24 & .84 \\
PO6 & 2.16 & .05 & 1.33 & 1.16 & .31 & .75 \\
PO7 & 2.67 & .06 & .81 & -.37 & .26 & .82 \\
\hline
\end{tabular}

Nota: $\mathrm{PA}=$ Pasión Armoniosa; $\mathrm{PO}=$ Pasión Obsesiva; Error estándar de la asimetría $=.08$; Error estándar de la curtosis $=.15$. Grados de libertad Kolmogorov-Smirnov y Shapiro-Wilk $=1,016$, todos los valores $p<.001$.

\section{Análisis factorial de la escala}

En esta investigación se puso a prueba la estructura factorial de la Escala de Pasión por el Trabajo de Vallerand et al. (2003) mediante el análisis confirmatorio de factores con ecuaciones estructurales con el método de estimación de máxima verosimilitud. Se analizó la estructura factorial siguiendo tres pasos. En primer lugar, se puso a prueba un modelo unifactorial (M1) donde los 14 ítems representan un factor latente único de pasión en el trabajo. Los resultados del análisis de factores confirmatorio del M1 no presentaron un buen ajuste para los datos (véase Tabla 2). En segundo lugar, se procedió a poner a prueba el modelo de dos factores (M2) compuesto por la pasión armoniosa y la pasión obsesiva. El M2 presentó un mejor ajuste para los datos que el M1 (véase Tabla 2). Sin embargo, no obtuvo índices de ajuste con niveles aceptables, según recomiendan varios expertos (Browne \& Cudeck, 1993). Luego de un análisis cualitativo de la composición de cada ítem, se determinó que tenían una sintaxis complicada. Como resultado de este análisis, se eliminaron los ítems 6 ("Este trabajo es una pasión que todavía logro controlar") y 9 ("El impulso es tan fuerte, que no puedo dejar de hacer este trabajo"). Según Kline (1986), la redacción de ítems debe ser lo más sencilla posible para lograr una interpretación acertada. Por último, se puso a prueba el modelo (M3) de dos factores de pasión por el trabajo sin incluir los ítems 6 y 9 (Figura 1).

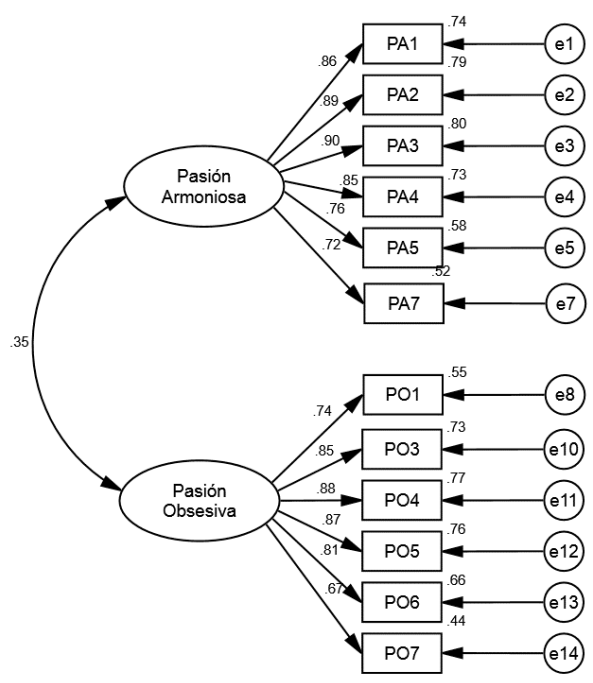

$\mathrm{CMIN}=452.371, \mathrm{DF}=53, \mathrm{p}=.000, \mathrm{AIC}=526.371, \mathrm{CFI}=.957, \mathrm{NFI}=.951, \mathrm{TLI}=.946, \mathrm{IFI}=.957$ RMSEA $=086$, PCLOSE $=000$

Figura 1: Modelo 3 (M3) de dos factores sin los items 6 y 9 de la Escala de Pasión por el Trabajo de Vallerand et al. (2003).

El M3 presentó un buen ajuste para los datos (véase Tabla 2). El análisis confirmatorio de factores demostró que el M3 posee mejor ajuste a los datos, $\chi^{2}=452.37$ (53) $p$ $<.001, R M S E A=.08, C F I=.95, N F I=.95, I F I=.96, A I C=$ $502.37, \chi^{2}$ corregido $=321.67(53) p<.001, C F I$ corregido $=$ .96, NFI corregido $=.93, I F I$ corregido $=.96$, AIC corregido $=453.68$. Los índices de ajuste relativos cumplen con los niveles aceptables propuestos por varios autores (Browne \& Cudeck, 1993; Satorra \& Bentler, 2001). Para comparar los modelos se utilizó el $A I C$ corregido debido a que todos los modelos difieren en el número de parámetros libres de estimación tales como el número de ítems y las covarianzas (AIC, Akaike, 1987).

Schumacker y Lomax (2010) recomiendan utilizar el AIC para comparar modelos de ecuaciones estructurales cuando los valores de $\chi^{2}$ son estadísticamente significativos. Debido a que los datos no siguen una distribución normal, se utilizó la corrección de Satorra y Bentler (2001) del AIC para comparar los tres modelos propuestos. El M3 presenta un índice menor $\left(A I C_{c o r r}=453.68\right)$ que los obtenidos por los otros dos modelos. Esto es indicativo de que el M3 tiene un mejor ajuste para los datos (véase de $\Delta A I C_{\text {corr }}$ en la Tabla 2). 
Tabla 2: Indicadores de ajuste para análisis confirmatorio de factores de la Escala de Pasión por el Trabajo

\begin{tabular}{|c|c|c|c|c|c|c|c|c|c|c|c|c|c|c|}
\hline Modelo & $x^{2}$ & $\chi_{\text {corr }}^{2}$ & $G L$ & RMSEA & CFI & $C F I_{\text {corr }}$ & $N F I$ & $N F I_{\text {corr }}$ & $I F I$ & $I F I_{\text {corr }}$ & $A I C$ & $A I C_{\text {corr }}$ & $\begin{array}{l}\triangle A I C_{\text {corr }} \\
\text { comparado } \\
\text { con } M 3\end{array}$ & $\begin{array}{c}\triangle R M S E A \\
\text { comparado con M. }\end{array}$ \\
\hline M1 & 5102.67 & 3564.85 & 77 & .25 & .57 & .57 & .37 & .49 & .57 & .57 & 5158.67 & 5104.31 & 4650.63 & .17 \\
\hline M2 & 953.38 & 672.92 & 76 & .10 & .92 & .93 & .92 & .88 & .92 & .93 & 1011.38 & 955.14 & 501.46 & .02 \\
\hline M3 & 452.37 & 321.67 & 53 & .08 & .96 & .96 & .95 & .93 & .96 & .96 & 502.37 & 453.68 & & \\
\hline
\end{tabular}

Nota: $\mathrm{M} 1=$ Modelo unifactorial; M2 = Modelo de dos factores; M3 = Modelo de dos factores sin ítems 6 y $9 ; \chi^{2}=$ chi cuadrado; $\chi_{\text {corr }}^{2}=$ chi cuadrado corregido; GL = grados de libertad; RMSEA = Root Mean Square Error Approximation; CFI = Comparative Fit Index; CFI corr $=$ Comparative Fit Index corregido; NFI = Normed Fit Index; NFI ${ }_{\text {corr }}=$ Normed Fit Index corregido; IFI = Incremental Fit Index; IFI ${ }_{\text {corr }}=$ Incremental Fit Index corregido; $\mathrm{AIC}=$ Akaike Information Criterion; $\triangle \mathrm{AIC}=$ Incremento; $\Delta \mathrm{RMSEA}=$ Incremento en RMSEA; Todos los estadísticos $\chi^{2}$ y $\chi_{\text {corr }}^{2}$ son significativos $p<.001$.

\section{Análisis de los ítems}

Se examinó la capacidad de discriminación de los ítems de la Escala de Pasión por el Trabajo a través del índice de correlación ítem total. Los mismos oscilaron entre .64 y .85, mientras que la varianza explicada en los ítems oscila entre .45 y .75 (véase Tabla 3 ). Los índices de discriminación están por encima del mínimo recomendado de .30 (Kline, 1986).

Tabla 3 Índices de discriminación y varianza explicada de los items de la Escala de Pasión por el Trabajo

\begin{tabular}{|c|c|c|}
\hline Ítems & $\begin{array}{c}\text { Índice de } \\
\text { discriminación }\end{array}$ & $R^{2}$ \\
\hline $\begin{array}{l}\text { 1. Este trabajo me permite vivir una } \\
\text { gran variedad de experiencias. }\end{array}$ & .80 & .71 \\
\hline $\begin{array}{l}\text { 2. Las cosas nuevas que aprendo con } \\
\text { este trabajo hacen que lo valore } \\
\text { todavía más. }\end{array}$ & .85 & .74 \\
\hline $\begin{array}{l}\text { 3. Este trabajo me permite vivir } \\
\text { experiencias memorables. }\end{array}$ & .85 & .75 \\
\hline $\begin{array}{l}\text { 4. Este trabajo refleja las cualidades } \\
\text { que valoro en mí. }\end{array}$ & .83 & .69 \\
\hline $\begin{array}{l}\text { 5. Este trabajo está en armonía con } \\
\text { otras actividades de mi vida. }\end{array}$ & .74 & .57 \\
\hline $\begin{array}{l}\text { 6. Estoy completamente implicado con } \\
\text { este trabajo. }\end{array}$ & .70 & .50 \\
\hline 7. No puedo vivir sin este trabajo. & .69 & .57 \\
\hline $\begin{array}{l}\text { 8. No puedo concebir mi vida sin este } \\
\text { trabajo. }\end{array}$ & .81 & .72 \\
\hline $\begin{array}{l}\text { 9. Dependo emocionalmente de este } \\
\text { trabajo. }\end{array}$ & .83 & .71 \\
\hline $\begin{array}{l}\text { 10. Me cuesta controlar la necesidad de } \\
\text { realizar este trabajo. }\end{array}$ & .82 & .71 \\
\hline $\begin{array}{l}\text { 11. Tengo un sentimiento casi obsesivo } \\
\text { por este trabajo. }\end{array}$ & .78 & .64 \\
\hline $\begin{array}{l}\text { 12. Mi estado de ánimo depende de mi } \\
\text { capacidad para realizar este trabajo. }\end{array}$ & .64 & .45 \\
\hline
\end{tabular}

Nota: $R^{2}=$ Varianza explicada; Pasión Armoniosa 1-6; Pasión Obsesiva $7-12$

\section{Análisis de fiabilidad}

Con el modelo final de dos factores de pasión por el trabajo, se procedió a examina-r la confiabilidad y la fiabilidad compuesta. Los valores alfa de Cronbach fluctuaron entre .75 y .94 . La fiabilidad compuesta de los constructos oscila entre .77 y .93. En la Tabla 4 se presentan los valores de fiabilidad compuesta para cada constructo.

\section{Análisis de validez discriminante}

Para evaluar la validez convergente y discriminante se calculó la Varianza Media Extraída (VME), la cual mide el nivel de varianza explicada por el constructo en los ítems. Mientras más alto el índice de VME, menor la varianza de error en los ítems. Los valores de VME de todos los constructos fluctuaron entre .50 y .79 (véase Tabla 4), por lo que se consideran aceptables (Fornell \& Larcker, 1981). Se procedió a examinar la relación entre los constructos. En la Tabla 4 se presentan las correlaciones entre los mismos. Las correlaciones entre los constructos fluctúan entre .05 y .77. La varianza compartida entre dos constructos siempre es menor a la varianza explicada por cada uno de ellos, lo cual cumple con el criterio de validez discriminante propuesto por Fornell y Larcker (1981).

\section{Discusión}

Este estudio tenía como objetivo determinar las propiedades psicométricas de la Escala de Pasión por el Trabajo traducida por Orgambidez-Ramos et al. (2014) y diferenciar el concepto de pasión por el trabajo de engagement en el trabajo, la centralidad del trabajo, la motivación intrínseca y el significado del trabajo. De acuerdo a los resultados obtenidos, podemos concluir que el instrumento es apropiado para ser usado en investigaciones $\mathrm{y}$ en intervenciones organizacionales.

Nuestros resultados confirman la estructura bi-factorial de la pasión por el trabajo (pasión armoniosa y pasión obsesiva) 
como la del mejor ajuste en el contexto puertorriqueño. Esta conclusión está a la par de investigaciones previas sobre las propiedades psicométricas en otros países, tales como Canadá (Donahue et al., 2012), Francia (Vallerand et al., 2010), Suecia (Thorgren et al., 2013), Rusia (Astakhova, 2015), Noruega (Donahue et al., 2012) y España (Chamarro et al., 2015).

En esta investigación, se eliminaron los ítems 6 y 9 de la escala debido al lenguaje y la estructura utilizada para la redacción. El ítem 6, "Este trabajo es una pasión que todavía logro controlar", contiene una premisa de ambigüedad sobre cuándo y cómo la persona logra controlar la pasión. Por otro lado, el ítem 9, "El impulso es tan fuerte, que no puedo dejar de hacer este trabajo", está redactado de forma compleja con dos características que busca medir: la fuerza del impulso y la frecuencia del impulso. Los ítems de la escala deben ser redactados con lenguaje sencillo y una construcción simple de un solo concepto para evitar un error de medición del constructo (Kline, 1986). La literatura demuestra modificaciones similares a la escala. Por ejemplo, en el estudio de Zito y Colombo (2017), la versión final tiene 13 reactivos porque eliminaron el ítem 7. Además, correlacionaron el ítem 5 con el 6 y el ítem 8 con el 9, para reducir el error de estimación y eliminar la redundancia semántica. Similarmente, en nuestro estudio, los ítems 6 y 9 presentaron complicaciones de ajuste al modelo. Por otro lado, se han utilizado versiones acortadas de la escala de 12 ítems y 6 ítems con propiedades psicométricas aceptables (Marsh et al., 2013; Trépanier et al., 2014; Vallerand et al., 2010). La escala resultante de este estudio con 12 ítems es de fácil manejo para futuras investigaciones y usos en las organizaciones.

Las correlaciones entre las sub-escalas de pasión por el trabajo y el resto de los conceptos fluctúan de una magnitud baja a una alta. La mayoría de los conceptos tienen correlaciones significativas porque existen superposiciones teóricas, no obstante son distintos empíricamente. El análisis de validez discriminante confirma que cada instrumento mide un concepto diferente, sin que exista redundancia entre ellos. Estos resultados indican que las escalas miden independientemente la inclinación hacia el trabajo que se disfruta (pasión por el trabajo), el estado mental positivo de realización en el trabajo (engagement en el trabajo), el nivel de importancia que tiene el trabajo en la vida (centralidad del trabajo), el impulso inherente de trabajar (motivación intrínseca) y la valencia del trabajo (significado del trabajo). Por lo tanto, la pasión por el trabajo es una aportación única a la literatura de la psicología ocupacional.

\section{Fortalezas y limitaciones}

En primer lugar, el muestreo del estudio original fue por disponibilidad por lo que no se pueden generalizar los resultados de los datos. Sin embargo, se contó con una muestra amplia de 1,016 participantes que facilitó la recopilación de información de diversos grupos demográficos. Además, los resultados coinciden con otros estudio sobre las propiedades de la Escala de Pasión por el Trabajo de Vallerand et al. (2003).

Para futuras investigaciones, se recomienda estudiar las relaciones de la pasión por el trabajo con otras variables individuales y organizacionales, como por ejemplo la adicción al trabajo, el compromiso organizacional y el desempeño. Además, se recomienda estudiar las propiedades psicométricas de la Escala de Pasión por el Trabajo de Vallerand et al. (2003) a través del tiempo.

\section{Implicaciones teóricas y prácticas}

Los resultados de esta investigación tienen implicaciones prácticas y teóricas importantes. En primer lugar, los resultados demuestran que la Escala de Pasión por el Trabajo de Vallerand et al. (2003) es un instrumento apropiado para investigaciones e intervenciones organizacionales en la fuerza laboral en Puerto Rico debido a que mide el concepto de forma válida y consistentemente. El uso de la escala permitirá estudiar las relaciones de la pasión por el trabajo con otras variables organizacionales y psicosociales para aportar nuevo conocimiento a la psicología ocupacional positiva. Al establecer la validez y fiabilidad del instrumento, nos aseguramos de utilizar medidas apropiadas en la fuerza laboral puertorriqueña. Por otro lado, la principal aportación a nivel teórico de nuestra investigación radica en que es de los pocos estudios que establece las diferencias entre la pasión por el trabajo y el engagement en el trabajo, la centralidad del trabajo, la motivación intrínseca y el significado del trabajo con evidencia empírica. Esta evidencia empírica sustenta los planteamientos teóricos (Curran et al., 2015; Lisbona et al., 2016; Perrewé et al., 2014) que establecen las diferencias entre la pasión y el resto de los constructos. En la literatura se establecen las diferencias conceptuales entre los constructos, pero no se presenta la evidencia empírica donde se avalen dichas diferencias y nuestro estudio contribuye en esa línea. La diferenciación de los constructos y su correspondiente evidencia empírica es esencial para preservar la parsimonia científica y facilitar la generación sistemática de conocimiento científico.

\section{Conclusiones}

La pasión por el trabajo es un concepto que tiene una trayectoria histórica amplia y fundamentada filosóficamente (Vallerand, 2015). En años recientes se ha demostrado que es un concepto clave para lograr un mejor entendimiento de cómo los procesos psicológicos propician resultados positivos o negativos (Donahue et al., 2012; Marsh et al., 2013). Por lo tanto, podemos confirmar que la pasión no es 
vino viejo en botella nueva, más bien es un vino de 2,000 años descubierto en una tablilla desatendida y listo para consumir en la actualidad.

\section{Referencias}

Akaike, H. (1987). Factor analysis and AIC. Psychometrika, $52,317-332$.

Ashforth, B. E., \& Mael, F. (1989). Social identity theory and the organization. Academy Of Management Review, 14(1), 20-39. doi:10.5465/AMR.1989.4278

Astakhova, M. N. (2015). The curvilinear relationship between work passion and organizational citizenship behavior. Journal of Business Ethics, 130(2), 361374. doi: $10.1007 / \mathrm{s} 10551-014-2233-5$

Birkeland, I. K., \& Buch, R. (2014). The dualistic model of passion for work: Discriminate and predictive validity with work engagement and workaholism. Motivation and Emotion, 39(3), 392-408. doi:10.1007/s11031014-9462-x

Browne, M.W. \& Cudeck, R. (1993). Alternative ways of assessing model fit. En Bollen, K.A. \& Long, J.S. (Eds.), Testing structural equation models (pp. 136162). Newbury Park, CA: Sage.

Chamarro, A., Penelo, E., Fornieles, A., Oberst, U., Vallerand, R. J., \& Fernández-Castro, J. (2015). Psychometric properties of the Spanish version of the Passion Scale. Psicothema, 27(4), 402-409. doi:10.7334/psicothema2015.80

Curran, T., Hill, A. P., Appleton, P. R., Vallerand, R. J., \& Standage, M. (2015). The psychology of passion: A meta-analytical review of a decade of research on intrapersonal outcomes. Motivation and Emotion, 39(5), 631-655. doi:org/10.1007/s11031-015-9503-0

Deci, E. L., \& Ryan, R. M. (1985) Intrinsic motivation and self-determination in human behavior. New York: Plenum.

Deci, E. L., \& Ryan, R. M. (2000). The "what" and "why" of goal pursuits: Human needs and the selfdetermination of behavior. Psychological Inquiry, 11(4), 227-268. doi:10.1207/S15327965PLI1104_01

Deci, E., Eghrari, H., Patrick, B. C., \& Leone, D. R. (1994). Facilitating internalization: The self-determination theory perspective. Journal of Personality, 62(1) doi:10.1111/j.1467-6494.1994.tb00797.x

Donahue, E. G., Forest, J., Vallerand, R. J., Lemyre, P.-N., Crevier-Braud, L., \& Bergeron, E. (2012). Passion for work and emotional exhaustion: the mediating role of rumination and recovery. Applied Psychology. Health and Well-Being, 4(3), 341-68. doi:10.1111/j.17580854.2012.01078.x

Doornik, J. A. and Hansen, H. (2008). An omnibus test for univariate and multivariate normality. Oxford Bulletin of Economics and Statistics, 70, 927-939. doi:10.1111/j.1468-0084.2008.00537.x

Duckworth, A. (2016). Grit: The power of passion and perseverance. New York: Scribner.

[EverySteveJobsVideo]. (2015, Marzo 17). Steve Jobs unveils the Think Different campaign (1997). [Video Archivo]. Recuperado de https://www.youtube.com/ watch? $=4 \mathrm{HsGAc} 0 \_$Y $5 \mathrm{c}$

Fernández-Ríos, L. (2008). Una revisión critica de la psicología positiva historia y concepto. Revista Colombiana de Psicología, 12(17), 161-178.

Fernández-Ríos, L., \& Novo, M. (2012). Positive pychology: Zeigeist (or spirit of the times) or ignorance (or disinformation) of history? International Journal of Clinical and Health Psychology, 12(2), 333-344.

Forest, J., Mageau, G. a., Sarrazin, C., \& Morin, E. M. (2011). "Work is my passion": The different affective, behavioural, and cognitive consequences of harmonious and obsessive passion toward work. Canadian Journal of Administrative Sciences / Revue Canadienne Des Sciences de l'Administration, 28(1), 27-40. doi:10.1002/cjas. 170

Fornell, C., \& Larcker, D. F. (1981). Evaluating structural equation models with unobservable variables and measurement error. Journal of Marketing Research, 18(2), 39-50.

Google. (2017, Marzo 21). Google trends. Recuperado de https://trends.google.com/ trends/explore?date=2007-02-21\%20 2017-03-21\&q=work\%20passion,find \%20your\%20 passion

Guay, F., Vallerand, R. J., \& Blanchard, C. (2000). On the assessment of situational intrinsic and extrinsic motivation: The situational motivation scale (SIMS). Motivation and Emotion, 24(3), 175-213. doi:10.1023/A:1005614228250

Ho, V. T., Wong, S.-S., \& Lee, C. H. (2011). A tale of passion: Linking job passion and cognitive engagement to employee work performance. Journal of Management Studies, 48(1), 26-47. doi:10.1111/ j.1467-6486.2009.00878.x 
Kline, P. (1986). A handbook of test construction: Introduction to psychometric design. New York:Methuen

Koestner, R., \& Losier, G. F. (2002). Distinguishing three ways of being highly motivated: A closer look at introjection, identification, and in- trinsic motivation. En E. L. Deci \& R. M. Ryan (Eds.), Handbook of selfdetermination research (pp. 101-121). Rochester, NY: The University of Rochester Press.

Lavigne, G. L., Forest, J., Fernet, C., \& Crevier-Braud, L. (2014). Passion at work and workers' evaluations of job demands and resources: a longitudinal study. Journal of Applied Social Psychology, 44(4), 255265. doi:10.1111/jasp.12209

Lazarus, R. S. (2003). Target article: Does the positive psychology movement have legs? Psychological Inquiry, 14(2), 93-109. doi:10.1207/ S15327965PLI1402_02

Le, H., Schmidt, F. L., Harter, J. K., \& Lauver, K. J. (2010). The problem of empirical redundancy of constructs in organizational research: An empirical investigation. Organizational Behavior and Human Decision Processes, 112(2), 112-125. doi:10.1016/j. obhdp.2010.02.003

Liu, R. (1997, Octubre 15). Apple reports wider loss. CNN Money. Recuperado de http://money.cnn. com/1997/10/15/companies/apple/

Lisbona-Bañuelos, A., Palací-Descals, F., \& BernabéCastaño, M. (2016). ¿Podemos apasionarnos por el trabajo?: Una revisión de la pasión para el trabajo. Papeles del Psicólogo, 37(3), 165-169.

Mageau, G. a., Vallerand, R. J., Charest, J., Salvy, S. J., Lacaille, N., Bouffard, T., \& Koestner, R. (2009). On the development of harmonious and obsessive passion: The role of autonomy support, activity specialization, and identification with the activity. Journal of Personality, 77(3), 601-646. doi:10.1111/ j.1467-6494.2009.00559.x

Marsh, H. W., Vallerand, R. J., Lafrenière, M.-A. K., Parker, P., Morin, A. J. S., Carbonneau, N., Paquet, Y. (2013). Passion: Does one scale fit all? Construct validity of two-factor passion scale and psychometric invariance over different activities and languages. Psychological Assessment, 25(3), 796-809. doi:10.1037/a0032573

Martínez-Lugo, M., Andújar-Rojas, C., \& RodriguezMontalbán, R. (2015). Escala breve de centralidad del trabajo. Manuscrito no publicado, Departamento de Psicología Industrial Organizacional, Universidad Albizu, San Juan, Puerto Rico.

MOW, International Research Team. (1987). The Meaning of Working. London: Academic Press.

Orgambídez-Ramos, A., Borrego-Alés, Y., \& Gonçalves, G. (2014). Passionate workers: A Spanish adaptation of the Passion Scale. Revista de Psicología Del Trabajo $y$ de Las Organizaciones, 30(2), 43-48. doi:10.1016/j. rpto.2014.06.005

Paullay, I. M., Alliger, G. M., \& Stone-Romero, E. F. (1994). Construct validation of two instruments designed to measure job involvement and work centrality. Journal of Applied Psychology, 79(2), 224-228.

Pérez-Álvarez, M. (2012). La psicología positiva: Magia simpática. Papeles del Psicólogo, 33(3), 183-201.

Perrewé, P. L., Hochwarter, W. A., Ferris, G. R., Mcallister, C. P., \& Harris, J. N. (2014). Developing a passion for work passion : Future directions on an emerging construct. Journal of Organizational Behavior, 35, 145-150. doi:10.1002/job

Rodriguez-Montalbán, R., Martínez-Lugo, M., Ortega, C., del Valle, A., Rosario, A., Gómez, C., Serrano, R. (2016, Noviembre). Amando lo que hago en mi trabajo: centralidad, autonomía, pasión y desempeño. Estudio presentado en Convención Nacional de Asociación de Psicología de Puerto Rico, San Juan, Puerto Rico.

Rodriguez-Montalbán, R., Martínez-Lugo, M., SánchezCardona, I. (2015). Escala del significado de la tarea. Manuscrito no publicado, Departamento de Psicología Industrial Organizacional, Universidad Albizu, San Juan, Puerto Rico.

Salanova, M., \& Schaufeli, W. (2009). El engagement en el trabajo: cuando el trabajo se convierte en pasión. Madrid: Alianza.

Satorra, A., \& Bentler, P. M. (2001). A scaled difference chi-square test statistic for moment structure analysis. Psychometrika, 66(4), 507-514. doi:10.1007/ BF02296192

Schaufeli, W. B., Bakker, A. B., \& Salanova, M. (2006). The measurement of work engagement with a short questionnaire: A cross-national study. Educational and Psychological Measurement, 66(4), 701-716. doi:10.1177/0013164405282471 
Schaufeli, W. B., Salanova, M., Bakker, A. B., \& GonzalesRoma, V. (2002). The measurement of engagement and burnout: A two sample confirmatory factor analytic approach. Journal of Happiness Studies, 3, 71-92. doi:10.1023/A:1015630930326

Schumacker, R. E., \& Lomax, R. G. (2010). A beginner's guide to structural equation modeling ( $3 \mathrm{rd} \mathrm{Ed}$.). Mahwah, NJ: Erlbaum.

Thorgren, S., Wincent, J., \& Sirén, C. (2013). The influence of passion and work-life thoughts on work satisfaction. Human Resource Development Quarterly, 24(4), 469-492. doi:10.1002/hrdq.21172

Trépanier, S., Fernet, C., Austin, S., Forest, J., \& Vallerand, R. (2014). Linking job demands and resources to burnout and work engagement: Does passion underlie these differential relationships? Motivation and Emotion, 38(3), 353-366.

Vallerand, R. (2015). The psychology of passion: A dualistic model. Nueva York: Oxford University Press.

Vallerand, R. J., Blanchard, C., Mageau, G. a, Koestner, R., Ratelle, C., Leonard, M., \& Marsolais, J. (2003). Les passions de l'ame: on obsessive and harmonious passion. Journal of Personality and Social Psychology, 85(4), 756-67. doi: 10.1037/0022-3514.85.4.756

Vallerand, R. J. \& Houlfort, N. (2003). Passion at work: Toward a new conceptualization. En S. W. Gilliand, D. D. Steiner, \& D. P. Skarlicki (Eds), Emerging perspectives on values in organizations (pp. 175204). North Carolina: Information Age Publishing.

Vallerand, R. J., Paquet, Y., Philippe, F. L., \& Charest, J. (2010). On the role of passion for work in burnout: A process model. Journal of Personality, 78(1), 289312. doi:10.1111/j.1467-6494.2009.00616.x

Wrzesniewski, A., Dutton, J. E., \& Debebe, G. (2003). Interpersonal sensemaking and the meaning of work. Research in Organizational Behavior, 25(January), 93-135.

Zito, M., \& Colombo, L. (2017). The Italian version of the passion for work scale: First psychometric evaluations. Journal of Work and Organizational Psychology, 33(1), 47-53. 